\title{
Usefulness of Maximal Expiratory Pressure in Evaluating Dysphagia after Ischemic Stroke
}

\author{
Bo Seong Jang, M.D., Ho Joong Jeong, M.D., Ph.D., Han Eum Choi, M.D., \\ Jae Hyun Lee, M.D., Young Joo Sim, M.D., Ph.D., Ghi Chan Kim, M.D., Ph.D. \\ Department of Physical Medicine and Rehabilitation, Kosin University Gospel Hospital, Busan, Korea
}

\begin{abstract}
Objective: We investigated the usefulness of maximal expiratory pressure (MEP) in evaluating dysphagia subsequent to ischemic stroke.

Methods: This study included patients with ischemic stroke who underwent MEP testing and videofluoroscopic swallowing study (VFSS), from October 2016 to February 2020. The VFSS findings were interpreted using the penetrationaspiration scale (PAS) and functional dysphagia scale (FDS). Patients were stratified into the non-aspiration ( $=59$ ) and aspiration $(n=47)$ groups. Partial correlation analysis among MEP, PAS, and FDS was performed after adjusting for age. Binary logistic regression using PAS was conducted to investigate the risk factors predisposing patients to inclusion in the aspiration group. Multiple linear regression using FDS was conducted to investigate the risk factors according to dysphagia severity. Receiver operating characteristic (ROC) curve analysis was applied to investigate factors which could be useful for detecting aspiration.

Results: Student's t-test revealed a significant difference in MEP between the non-aspiration and aspiration groups. MEP showed a positive correlation with PAS and FDS. MEP was also determined to be a risk factor for inclusion into the aspiration group, and a risk factor according to the severity of dysphagia. In the ROC curve analysis, MEP showed good diagnostic properties to help classify patients with aspiration.

Conclusion: Our results indicate that swallowing assessment can predict and help prevent aspiration pneumonia in patients with ischemic stroke. In the present study, MEP showed significant association with aspiration and the severity of dysphagia. Thus, determining the MEP during swallowing assessment in patients with ischemic stroke is potentially a useful parameter to predict dysphagia. (JKDS 2021;11:59-66)
\end{abstract}

Keywords: Maximal expiratory pressure, Dysphagia, Stroke

\section{INTRODUCTION}

Stroke is one of the most common causes of swallowing difficulty and induces neurological damage in various structures of the brain, leading to dysfunction of the oral cavity, pharynx, and esophagus ${ }^{1}$.
As a result, aspiration is frequently observed in stroke patients and can cause serious complications such as aspiration pneumonia. Therefore, it is crucial to diagnose dysphagia and conduct appropriate rehabilitation procedures to attain a better prognosis ${ }^{2,3}$. Respiratory muscles play a key role in protecting
Received: July 29 2020, Revised: August 4 2020,

Accepted: September 152020

Corresponding author: Ho Joong Jeong, Department of Physical Medicine and Rehabilitation, Kosin University College of Medicine, 262 Gamcheon-ro, Seo-gu, Busan 49267, Korea Tel: +82-51-990-6156, Fax: +82-51-990-3181

E-mail: jhjpmr@naver.com
Copyrights (c) The Korean Dysphagia Society, 2021. 
the airway. Various clinical indicators could be measured by evaluating respiratory muscles; for example, maximal expiratory pressure (MEP) and maximal inspiratory pressure (MIP) reflect the maximal strength of the respiratory muscles and are generated during expiration and inspiration, respectively. Coughing, an airway-protective mechanism to avoid aspiration, occurs through the rapid contraction of the diaphragm and external intercostal muscles ${ }^{4,5}$. In the clinical setting, the peak cough flow (PCF) is usually adopted to measure the maximal airflow generated during a cough. Pulmonary function parameters such as lung volume, flow rate, and capacity can be measured using a pulmonary function test (PFT).

Patients with stroke can have accompanying central diaphragmatic dysfunction and decreased respiratory muscle motion, resulting in various impairments in respiratory function. In previous studies, stroke patients demonstrated reduced respiratory muscle strength parameters, including MEP and MIP, and inhibited pulmonary function parameters, including forced vital capacity (FVC) and forced expiratory volume in $1 \mathrm{~s}$ (FEV1), relative to the healthy control group ${ }^{6}$. Stroke patients with dysphagia have also been reported to have reduced FVC and FEV1 compared with stroke patients without dysphagia? Other studies reported that stroke patients with dysphagia showed reduced coughing ability compared with stroke patients without dysphagia, and that PCF was a useful factor for evaluating the risk of aspiration ${ }^{8,9}$. Further, several studies have been published on the association among pulmonary function, coughing ability, and stroke-induced dysphagia. However, few studies to date have examined the association between respiratory muscle strength and stroke-induced dysphagia. Moreover, as coughing shares anatomical structures with expiration, it is valuable to evaluate the association between PCF and MEP.

Given these considerations, this study aimed to investigate the association among respiratory muscle strength, pulmonary function, coughing ability, and dysphagia after stroke.

\section{MATERIALS AND METHODS}

A retrospective review of the medical charts of patients who were diagnosed with acute ischemic hemispheric stroke at Kosin University Gospel Hospital between October 2016 and February 2020 was performed. The inclusion criteria were (1) a diagnosis of acute ischemic hemispheric stroke based on neurological examination and brain magnetic resonance imaging; (2) available data from a videofluoroscopic swallowing study (VFSS) performed within 3 weeks after the onset of acute ischemic hemispheric stroke; (3) measurement data of MIP, MEP, PCF, PFT, and the Korean version of the modified Barthel index (K$\mathrm{MBI}$ ). Because the incidence of dysphagia may vary depending on the stroke lesion, we selected only patients who experienced acute ischemic hemispheric stroke in the middle cerebral artery territory. The exclusion criteria were (1) hemorrhagic stroke or history of previous stroke; (2) tracheostomy; (3) inability to undergo MIP, MEP, PFT, or PCF assessment; and (4) history of cardiopulmonary disease. The sample size based on the area under receiver operating characteristic (ROC) curve of PAS was estimated using MedCalc (MedCalc Software, Ostend, Belgium), with a power of 0.90 and a significance level of 0.01 . The null hypothesis value was set at 0.5 .

\section{Maximal expiratory pressure and maximal inspiratory pressure}

The patients wore a flanged silicone mouthpiece, and a rehabilitation physician measured the MEP and MIP using a micro respiratory pressure meter (Carefusion, San Diego, CA, USA). Before testing, the patients were instructed to adapt to using the device to ensure more accurate measurements. Tests were performed with the patients wearing a nose clip, in the sitting position with the legs and trunk supported ${ }^{10}$. Two tests with a 5-min interval were performed to evaluate the maximal respiratory strength, and the higher of the two measurement values was chosen for analysis. 


\section{Pulmonary function test and peak cough flow test}

PFT was performed by a respiratory technician using a spirometer. The FVC and FEV1 were measured in this test. A PCF test was conducted by the same technician using a Personal Best flow meter (Philips Respironics Inc., Murrysville, PA, USA). PCF testing was performed two times with a 5-min interval to evaluate the maximal airflow during coughing, and the higher of the two measurement values was adopted for further analysis. PFT and PCF testing were performed with the patient in the same position as during the evaluation of MEP and $\mathrm{MIP}^{11}$.

\section{Videofluoroscopic swallowing study}

VFSS was performed by an experienced radiation technician and involved barium-impregnated boluses in the order of $5 \mathrm{~mL}$ thin liquid, pudding, porridge, and rice. Testing was stopped if a large amount of direct aspiration was observed or if delayed aspiration due to residual volume occurred. The testing procedure was video recorded, and two trained rehabilitation physicians interpreted the video clips. If the two rehabilitation physicians had different interpretations, the data from the more experienced physician were adopted. The VFSS results were interpreted according to the penetration-aspiration scale (PAS) and videofluoroscopic functional dysphagia scale $(\mathrm{FDS})^{12,13}$. A total score of up to 8 points is possible on the PAS, and each score was determined according to the presence or absence of aspiration, presence or absence of penetration, and level of airway invasion. Patients with no penetration or aspiration were awarded 1 point, those with penetration were awarded from 2 to 5 points, and those with aspiration were awarded 6 to 8 points. Following a previous study involving the PAS, patients with scores of 1 to 5 points were stratified into a non-aspiration group and those with scores of 6 to 8 points were stratified into an aspiration group ${ }^{14}$. A total score of up to 100 points is possible on the FDS, and each score was determined in the oral and pharyngeal phases. The closer the FDS score is to 100 points, the greater the severity of dysphagia. The functional oral intake scale was also evaluated based on the feeding type at the time of VFSS ${ }^{15}$.

\section{Korean version of the modified Barthel index}

In a previous study, the $\mathrm{K}-\mathrm{MBI}$ was used to measure the patients' general function ${ }^{14}$. In this study, we also adopted the K-MBI to measure the patients' activities of daily living.

\section{Statistical analysis}

Pearson correlation analysis was conducted to compare the VFSS results with the MEP, MIP, PCF, K-MBI, FVC, FEV1, and FEV1/FVC. Partial correlation analysis was conducted to compare the VFSS results with the factors that had a correlation in Pearson correlation analysis, considering age as a confounding factor. Student's t-test was performed to compare the MEP, MIP, PCF, K-MBI, FVC, FEV1, FEV1/FVC, and FDS values between the non-aspiration and aspiration groups. Binary logistic regression analysis using PAS was conducted to elucidate the risk factors for inclusion into the aspiration group. The forward conditional method was used for binary logistic regression analysis. Multiple linear regression analysis using FDS was conducted to elucidate the risk factors according to the severity of dysphagia. The stepwise method was used for multiple linear regression analysis. The independent factors used in binary logistic regression and multiple linear regression analyses were MEP, PCF, MIP, K-MBI, age, FVC, FEV1, and FEV1/FVC. Using ROC curve analysis, we investigated which factor could be adopted as a screening tool for detecting aspiration. The thresholds for the MEP and PCF values were calculated to determine the sensitivity and specificity. The SPSS version 21.0 software program (IBM, Armonk, NY, USA) was used for statistical analysis, and the significance level was set at $\mathrm{P}<0.05$. 


\section{RESULTS}

Out of the eligible 140 patients, 34 patients were excluded according to the exclusion criteria. Finally, 106 patients were included in this study (59 patients in the non-aspiration group and 47 patients in the aspiration group). The minimum required sample size

Table 1. Demographics, clinical factors, and baseline evaluation of stroke patients according to absence or presence of aspiration.

\begin{tabular}{lccc}
\hline & $\begin{array}{c}\text { Non-aspiration } \\
(\mathrm{n}=59)\end{array}$ & $\begin{array}{c}\text { Aspiration } \\
(\mathrm{n}=47)\end{array}$ & P-value \\
\hline Age (years) & $70.9 \pm 9.0$ & $71.8 \pm 12.5$ & $0.048^{*}$ \\
Sex & $39(66 \%)$ & $25(53 \%)$ & \\
$\quad$ Male & $20(34 \%)$ & $22(47 \%)$ & 0.183 \\
$\quad$ Female & $55.1 \pm 25.4$ & $23.2 \pm 9.2$ & $<0.001^{* * * *}$ \\
MEP (cmH $\left.\mathrm{CH}_{2} \mathrm{O}\right)$ & $31.6 \pm 16.6$ & $16.6 \pm 5.8$ & $<0.001^{* * * *}$ \\
MIP (cmH $\left.\mathrm{H}_{2} \mathrm{O}\right)$ & $224.9 \pm 83.0$ & $107.4 \pm 36.2$ & $<0.001^{* * * *}$ \\
PCF (L/min) & $2.44 \pm 0.94$ & $2.15 \pm 0.95$ & 0.109 \\
FVC (L) & $1.87 \pm 0.75$ & $1.57 \pm 0.69$ & 0.114 \\
FEV1 (L) & $0.77 \pm 0.10$ & $0.74 \pm 0.10$ & 0.168 \\
FEV1/FVC & $65.8 \pm 25.4$ & $50.9 \pm 30.2$ & $0.008^{* * *}$ \\
K-MBI & $14.9 \pm 15.8$ & $43.5 \pm 17.3$ & $<0.001^{* * * * *}$ \\
FDS & $2.59 \pm 1.22$ & $2.57 \pm 1.36$ & 0.941 \\
FOIS & & & \\
Stroke laterality & $32(54 \%)$ & $22(47 \%)$ & 0.453 \\
$\quad$ Left & $27(46 \%)$ & $25(53 \%)$ & 0.452 \\
$\quad$ Right & $17.2 \pm 8.9$ & $21.2 \pm 9.1$ & $0.025^{* *}$ \\
NIHSS & & & \\
Medical history & $29(49 \%)$ & $27(57 \%)$ & 0.395 \\
$\quad$ HTN & $18(31 \%)$ & $14(30 \%)$ & 0.936 \\
DM & $17(28 \%)$ & $22(46 \%)$ & 0.069 \\
Smoking & & &
\end{tabular}

Values are presented as mean \pm standard deviation or number (\%). MEP: maximal expiratory pressure, MIP: maximal inspiratory pressure, PCF: peak cough flow, FVC: forced vital capacity, FEV1: forced expiratory volume in $1 \mathrm{~s}, \mathrm{~K}-\mathrm{MBI}$ : Korean version of the modified Barthel index, FDS: functional dysphagia scale, FOIS: functional oral intake scale, NIHSS: National Institutes of Health Stroke Scale, HTN: hypertension, DM: diabetes mellitus. ${ }^{*} \mathrm{P}<0.05,{ }^{* *} \mathrm{P}<0.01,{ }^{* * *} \mathrm{P}<0.001$ by Student's t-test. calculated using MedCalc was 52 patients. In Student's t-test, FVC, FEV1, and FEV1/FVC did not show a significant difference between the nonaspiration and aspiration groups. Age, MEP, MIP, PCF, K-MBI, FDS, and National Institutes of Health Stroke Scale showed a significant difference between the non-aspiration and aspiration groups.(Table 1) In partial correlation analysis, MEP, MIP, PCF, and KMBI showed negative correlations with PAS and FDS after adjusting for age.(Table 2) In binary logistic regression, decrease in MEP and PCF were eligible risk factors suggesting inclusion into the aspiration group.(Table 3) In multiple linear regression analysis, MEP and PCF were useful factors predicting aspiration according to the dysphagia severity.(Table 4) In the ROC curve analysis, MEP and PCF seemed to be useful parameters for classifying patients at a risk of aspiration.(Fig. 1) The MEP had an area under the

Table 2. Correlation between VFSS results and MEP, MIP, PCF, FVC, FEV1, and K-MBI.

\begin{tabular}{lll}
\hline & \multicolumn{1}{c}{ PAS } & \multicolumn{1}{c}{ FDS } \\
\hline MEP & $-0.528^{* * *}$ & $-0.561^{* * *}$ \\
MIP & $-0.558^{* * *}$ & $-0.477^{* * *}$ \\
PCF & $-0.511^{* *}$ & $-0.472^{* *}$ \\
FVC & -0.142 & -0.115 \\
FEV1 & -0.185 & -0.178 \\
K-MBI & $-0.436^{* *}$ & $-0.250^{*}$ \\
\hline
\end{tabular}

Values are presented as correlation coefficients.

VFSS: videofluoroscopic swallowing study, PAS: penetrationaspiration scale, FDS: functional dysphagia scale, MEP: maximal expiratory pressure, MIP: maximal inspiratory pressure, PCF: peak cough flow, FVC: forced vital capacity, FEV1: forced expiratory volume in $1 \mathrm{~s}$, K-MBI: Korean version of the modified Barthel index.

${ }^{*} \mathrm{P}<0.05,{ }^{* *} \mathrm{P}<0.01$ by partial correlation analysis after adjustment for age.

Table 3. Binary logistic regression analysis of risk factors dictating inclusion into the aspiration group.

\begin{tabular}{lccccc}
\hline Independent variable & $\beta$ & SE & P-value & OR & 95\% CI \\
\hline Decrease in MEP & -0.192 & 0.062 & $0.002^{* *}$ & 1.212 & $1.007-1.370$ \\
Decrease in PCF & -0.039 & 0.012 & $0.001^{* *}$ & 1.040 & $1.015-1.064$ \\
\hline
\end{tabular}

MEP: maximal expiratory pressure, PCF: peak cough flow, $\beta$ : regression coefficient, SE: standard error, OR: odds ratio, CI: confidence interval.

*** $\mathrm{P}<0.01,{ }^{*} * * * \mathrm{P}<0.001$. 
Table 4. Multiple linear regression analysis of risk factors according to dysphagia severity as assessed using FDS.

\begin{tabular}{|c|c|c|c|c|c|}
\hline Independent variable & $\beta$ & $\mathrm{P}$-value & $95 \% \mathrm{CI}$ & $\mathrm{R}^{2}$ & VIF \\
\hline MEP & -0.423 & $<0.001^{* * * k}$ & $-0.532--0.193$ & 0.336 & 5.676 \\
\hline PCF & -0.218 & $0.031^{*}$ & $-0.102--0.005$ & & 1.677 \\
\hline
\end{tabular}

FDS: functional dysphagia scale, MEP: maximal expiratory pressure, PCF: peak cough flow, $\beta$ : regression coefficient, CI: confidence interval, VIF: variance inflation factor.

$* \mathrm{P}<0.05,{ }^{* * * *} \mathrm{P}<0.001$
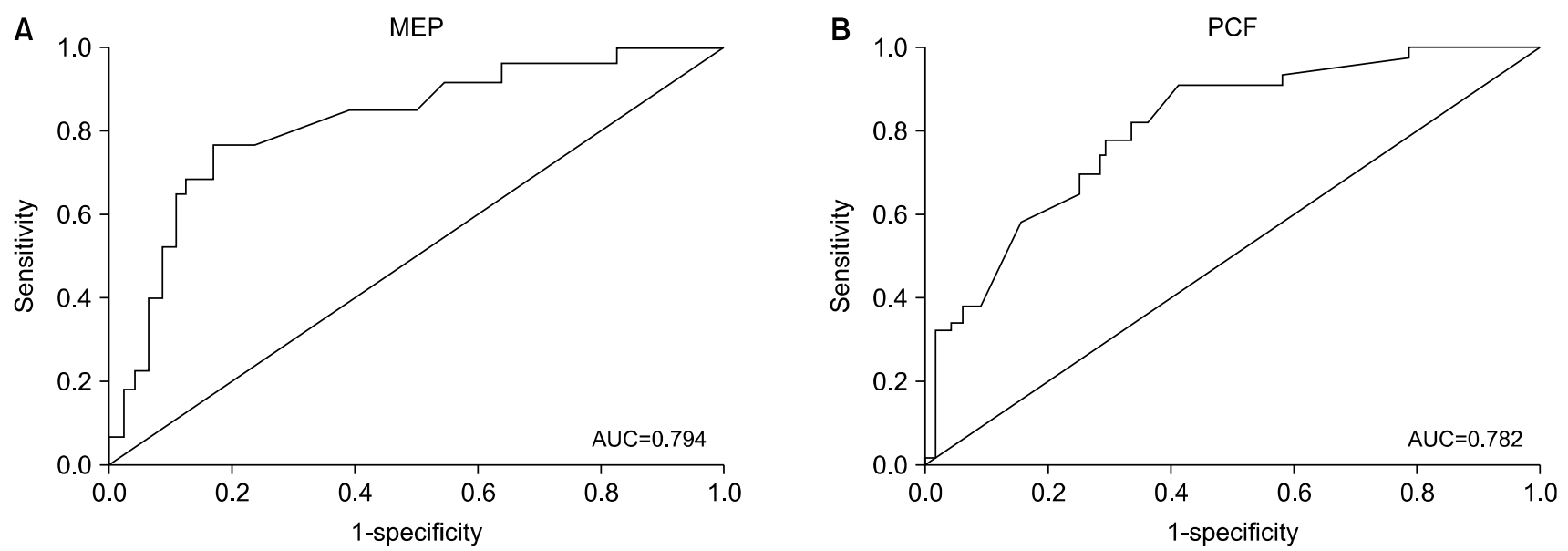

Fig. 1. Receiver operating characteristic curve and area under the curve (AUC) for detecting aspiration. (A) Maximal expiratory pressure (MEP). (B) Peak cough flow (PCF).

curve (AUC) of 0.794 (95\% confidence interval [CI]: $0.766-0.868$ ), with a sensitivity and specificity of $78.1 \%$ and $81.8 \%$, respectively, and a cutoff value of 33.50 $\mathrm{cmH}_{2} \mathrm{O}$. The PCF had an AUC of 0.782 (95\% CI: 0.6880.878 ), with a sensitivity and specificity of $79.6 \%$ and $74.4 \%$, respectively, and a cutoff value of $145 \mathrm{~L} / \mathrm{min}$.

\section{DISCUSSION}

In this study, low MEP and PCF values were found to be risk factors suggesting inclusion into the aspiration group, as well as risk factors according to the severity of dysphagia. MEP and PCF were useful clinical factors for detecting aspiration. Though the MIP and $\mathrm{K}-\mathrm{MBI}$ showed significant correlations with PAS and FDS, they failed to show increased risk for aspiration. No significant differences were observed in the FVC and FEV1 values between the non-aspiration and aspiration groups, and these parameters showed no association with aspiration.
MEP and MIP reflect respiratory muscle strength. Respiratory muscle training is known to improve respiratory muscle function and coughing, and to reduce the incidence of chest infection ${ }^{16}$. Another study suggested that respiratory muscle training reduces dysphagia in stroke patients ${ }^{17}$. In the present study, the mean values of MIP and MEP showed statistically significant differences between the nonaspiration and aspiration groups. In particular, MEP was observed to be a risk factor suggesting inclusion into the aspiration group and a risk factor according to the dysphagia severity. MEP was correlated with PAS and FDS, and was also identified as a useful clinical factor for detecting aspiration. Among various available mechanisms to prevent dysphagia, swallow apnea occurs in the pharyngeal phase of swallowing. Swallow apnea occurs during exhalation and, after swallowing, exhalation is maintained to increase the subglottic pressure to prevent the aspiration of food. At this time, the expiratory muscles become 
involved in maintaining exhalation. Therefore, MEP is considered a risk factor for aspiration because it is an indicator of the ability to maintain exhalation. Conversely, according to our present results, MIP may not be considered as a risk factor for aspiration because it plays minimal role in maintaining exhalation to prevent aspiration.

MEP is a useful factor that can be relatively easily measured in the clinical setting, and its usefulness had been confirmed in this study. However, the cutoff value of MEP in our study was too low relative to that for healthy people, and gold standard measures, such as PAS and FDS, are already available for swallowing assessment ${ }^{18}$. Further, a previous study had shown the effectiveness of respiratory muscle training for preventing aspiration pneumonia ${ }^{16}$. Therefore, we focused on the changes of aspiration according to the MEP value from a therapeutic point of view, not only from a diagnostic perspective for aspiration detection. Further studies are needed on how changes in aspiration are observed according to changes in MEP through respiratory muscle strengthening. MEP can be a useful indicator of the treatment effect of dysphagia rehabilitation.

In the present study, the average PCF value was higher in the non-aspiration group than in the aspiration group, and PCF had a statistically significant correlation with PAS and FDS. Moreover, PCF was observed as a risk factor suggesting inclusion into the aspiration group. A previous study found an apparent significant difference in PCF between stroke patients with and without dysphagia ${ }^{8}$. Further, PCF demonstrated a degree of clinical importance in the evaluation of dysphagia in patients with ischemic stroke in a previous study?. Coughing is a protective mechanism for keeping the airway clear, which may reduce the risk of aspiration pneumonia. Coughing shares anatomical structures with and is closely related to maximal respiratory pressure. PCF was also a useful factor for swallowing assessment in the present study. Therefore, further studies on the changes of aspiration according to the changes of PCF value are also needed, and PCF can also be a useful indicator of the treatment effect of dysphagia rehabilitation.

In the present study, a negative correlation was found between K-MBI and PAS, consistent with a previous study ${ }^{19}$. However, no significant difference in $\mathrm{K}-\mathrm{MBI}$ outcomes was found between the nonaspiration and aspiration groups. Further, K-MBI was not a risk factor suggesting inclusion into the aspiration group. Broadley et al. contended that a modified Barthel index score of $<20$ points was a risk factor for prolonged dysphagia ${ }^{20}$. In this study, $\mathrm{K}-\mathrm{MBI}$ was measured to check the activities of daily living of patients with ischemic hemispheric stroke, and it showed a significant correlation with PAS and FDS. However, as the mean values of K-MBI in the non-aspiration and aspiration groups were much higher than 20 points, it was difficult to confirm $\mathrm{K}-\mathrm{MBI}$ as a risk factor for dysphagia. Our results, however, need to be interpreted with caution since those who were too fragile and neurologically impaired to undergo VFSS with MBI values may have been excluded by the study design. Though sufficient level of cognition and cooperation is warranted to measure the MEP in these patients, future studies on the role of MEP values including those with low MBI values are warranted.

In patients with stroke, the diaphragm, intercostal muscles, and abdominal muscles become weakened and the contribution of thoracic movements during breathing is lower than that in healthy people, resulting in a decreased lung volume ${ }^{21-23}$. In this study, no significant differences in FVC and FEV1 were found between the non-aspiration and aspiration groups. Moreover, dysphagia was not a risk factor dictating inclusion into the aspiration group. One previous study found that reduced pulmonary function could increase the risk of aspiration in patients with stroke ${ }^{24}$. However, that study was conducted among patients with all types of stroke, whereas our study included homogeneous patients with ischemic hemispheric stroke in the middle cerebral artery territory. Therefore, it is possible that the effect of pulmonary function on aspiration could 
be different depending on the lesion and type of stroke involved. Further research according to the specific type and lesion of stroke could be helpful.

Several studies have been published on the effect of pulmonary function and the ability of cough to mitigate dysphagia. The previous study on the association between dysphagia and respiratory muscle strength in stroke patients performed a simple comparison of the average value of respiratory muscle strength, whereas the present study calculated the odds ratio through regression analysis and obtained the cutoff value through ROC curve analysis. This study is meaningful in that it comprehensively investigated which factor could be useful for predicting aspiration, considering respiratory muscle strength along with existing data on pulmonary function and the coughing ability. In our study, MEP and PCF were found to be useful clinical factors for detecting aspiration.

This study had several limitations. First, previous studies suggested that non-dominant hemispheric cortical lesions show a strong correlation with dysphagia $^{25,26}$. However, we did not stratify the patients into dominant and non-dominant hemispheric lesions. Our study would have been more meaningful had we divided the patients into dominant and non-dominant hemispheric lesions. Second, the VFSS results were interpreted with stratification into the non-aspiration and aspiration groups using PAS. Meanwhile, penetration was not independently considered in this study; instead, the non-aspiration group included patients with penetration. Overall, the number of patients with penetration was too small to establish an accurate representation of penetration. Therefore, the participants could not be divided into the nonaspiration, penetration, and aspiration groups. Future prospective studies involving a large sample size of patients could help determine if MEP values could also indicate risk of penetration.

The MEP is an index commonly used in the clinical setting for evaluating respiratory muscle strength. Preventing and predicting aspiration pneumonia through a VFSS is very important. In the present study, MEP was identified as a risk factor dictating inclusion into the aspiration group and it had an association with the severity of dysphagia. Therefore, evaluation of the MEP during the swallowing assessment may be of diagnostic values in patients with acute ischemic hemispheric stroke.

\section{CONFLICT OF INTEREST}

The authors declare that they have no conflict of interest.

\section{RESEARCH ETHICS}

Research Involving Human Participants: the study was reviewed and approved by the appropriate ethics committee(s). Informed Consent: This is a retrospective study with chart review. IRB approval number: Kosin university gospel hospital IRB 2019-05023.

\section{ACKNOWLEDGEMENTS}

There are no financial conflicts of interest to disclose.

\section{REFERENCES}

1. Marlis GF, Lauren O, Levan A, Asare BC. Dysphagia after Stroke: an Overview. Curr Phys Med Rehabil Rep. 2013;1:187-96.

2. Ramsey C, Smithard DG, Kalra L. Early assessments of dysphagia and aspiration risk in acute stroke patients. Stroke. 2003;34;1252-7.

3. Doggett DL, Tappe KA, Mitchell MD, Chapell R, Coates V. Dysphagia prevention of pneumonia in elderly stroke patients by systematic diagnosis and treatment of dysphagia: an evidence based comprehensive analysis of the literature. Dysphagia. 2001;16:279-95.

4. Hammond CAS, Goldstein LB, Horner RD, Ying J, Gray L, Gonzalez-Rothi L, et al. Predicting aspiration in patients with ischemic stroke: comparison of clinical signs and aerodynamic measures of voluntary cough. Chest. 2009; 135:769-77.

5. Pitts T, Morris K, Lindsey B, Davenport P, Poliacek I, Bolser D. Co-ordination of cough and swallow in vivo and in silico. Exp Physiol. 2012;97:469-73. 
6. Lima IN, Fregonezi GA, Rodrigo M, Cabral EE, Aliverti A, Campos TF, et al. Acute effects of volume-oriented incentive spirometry on chest wall volumes in patients after a stroke. Respir Care. 2014;59:1101-7.

7. Park GY, Kim SR, Kim YW, Jo KW, Lee EJ, Kim YM, et al. Decreased diaphragm excursion in stroke patients with dysphagia as assessed by M-mode sonography. Arch Phys Med Rehabil. 2015;96:114-21.

8. Kimura Y, Takahashi M, Wada F, Hachisuka K. Differences in the peak cough flow among stroke patients with and without Dysphagia. J UOEH. 2013;35:9-16.

9. Min SW, Oh SH, Kim GC, Sim YJ, Kim DK, Jeong HJ. Clinical importance of peak cough flow in dysphagia evaluation of patients diagnosed with ischemic stroke. Ann Rehabil Med. 2018;42:798-803.

10. Dimitriadis Z, Kapreli E, Konstantinidou I, Oldham JA, Strimpakos N. Test/retest reliability of maximum mouth pressure measurements with the MicroRPM in healthy volunteers. Respir Care. 2011;56:776-82.

11. Kang SW, Shin JC, Park CI, Moon JH, Rha DW, Cho DH. Relationship between inspiratory muscle strength and cough capacity in cervical spinal cord injured patients. Spinal Cord. 2006;44:242-8.

12. Rosenbek JC, Robbins JA, Roecker EB, Coyle JL, Wood JL. A penetration aspiration scale. Dysphagia. 1996;11: 93-8.

13. Han TR, Paik NJ, Park JW. Quantifying swallowing function after stroke: A functional dysphagia scale based on videofluoroscopic studies. Arch Phys Med Rehabil. 2001; 82:677-82.

14. Kim SB, Lee SJ, Lee KW, Lee JH, Kim DW. Usefulness of early videofluoroscopic swallowing study in acute stroke patients with dysphagia. Ann rehabil med. 2018;42:4251.

15. Crary MA, Carnaby-Mann GD, Groher ME. Initial psychometric assessment of a functional oral intake scale for dysphagia in stroke patients. Arch Phys Med Rehabil. 2005;86:1516-20.

16. Ross D Pollock, Ged F Rafferty, John Moxham, Lalit
Kalra. Respiratory muscle strength and training in stroke and neurology: a systematic reivew. Int J Stroke. 2013; 8:124-30.

17. Liaw MY, Hsu CH, Leong CP, Liao CY, Wang LY, Lu CH et al. Respiratory muscle training in stroke patients with respiratory muscle weakness, dysphagia, and dysarthria a prospective randomized trial. Medicine. 2020;99:e19337.

18. Leo FB, Robert EH. Maximal respiratory pressure: normal values and relationship to age and sex. Am Rev Respir Dis. 1969;99:696-702.

19. Kim DS, Sim YJ, Kim CC, Jeong HJ. The relationship between swallowing disorder and physical function in stroke. Kosin Med J. 2010;25:110-6.

20. Broadley S, Croser D, Cottrell J, Creevy M, Teo E, Yiu D, et al. Predictors of prolonged dysphagia following acute stroke. J Clin Neurosci. 2003;10:300-5.

21. Khedr EM, Shinawyb OE, Khedrc T, Alid YAA, Awad EM. Assessment of corticodiaphragmatic pathway and pulmonary function in acute ischemic stroke patients. Eur J Neurol. 2000;7:323-30.

22. Lanini B, Bianchi R, Romagnoli I, Coli C, Binazzi B, Gigliotti F, et al. Chest wall kinematics in patients with hemiplegia. Am J Respir Crit Care Med. 2003;168:10913.

23. Lima IN, Fregonezi GA, Rodrigo M, Cabral EE, Aliverti A, Campos TF, et al. Acute effects of volume-oriented incentive spirometry on chest wall volumes in patients after stroke. Respir Care. 2014;59:1101-7.

24. Yang HE, Park YK, Kim SM. Correlation between respiratory function and dysphagia in stroke patients. J Korean Dysphagia Soc. 2014;4:23-7.

25. Barer DH. The natural history and functional consequences of dysphagia after hemispheric stroke. J Neurol, Neurosurg, and Psychiatry.1989;52:236-41.

26. Schroeder MF, Daniels SK, McClan M, Corey DM, Foundas AL. Clinical and cognitive predictors of swallowing recovery in stroke. J Rehabil Res Dev.2006;43: 301-10. 